\title{
Voltage Reversal in Unbalanced Rectenna Association
}

\author{
Nicolas Degrenne, Vlad Marian, Christian Vollaire, François Buret, Jacques Verdier, and Bruno Allard
}

\begin{abstract}
Rectifying antenna (rectenna) associations can be advantageously used to harvest electromagnetic energy from different directions. When the rectennas are associated in series, their output voltages are added, and the electrical dc energy is more easily exploitable. This letter shows that if a rectenna receives significantly less energy than the others (i.e., unbalanced association), its voltage polarity may reverse. Simulation and experimental results show that when an unbalanced association is operated at maximum power point, voltage reversal can result in a total decrease in efficiency of up to $26 \%$. Possible solutions to reduce these detrimental consequences are also discussed.
\end{abstract}

Index Terms-Energy harvesting, rectenna, series association.

\section{INTRODUCTION}

$\mathbf{T}$ ODAY'S electronic devices have become increasingly portable and ubiquitous. There is an increasing demand for low-power autonomous sensors to monitor a wide range of parameters, from vital signs to temperature, luminosity, and air quality. Most of these devices rely on batteries for power, which represents a setback in the way of system miniaturization and the decrease in per-unit cost. In addition, battery disposal into the environment is an important source of pollution, and battery recycling is a complex and costly process.

Recent developments in low-density wireless energy transfer have shown that under certain conditions, a low-power system can rely solely on incident electromagnetic waves for power [1]. The electromagnetic power source can be either an emitter designed for voluntary power transmission in its vicinity [2] (point to point or broadcasting) or even ambient electromagnetic radiation due to wireless communication networks like WLAN and the various cellular telephone standards [3].

A rectifying antenna (rectenna) is the transducer capable of converting high-frequency electromagnetic waves into dc electrical energy. It is the main component of an electromagnetic energy receiver.

When considerable energy is needed or when harvesting energy from the ambient electromagnetic field, the use of a single rectenna is often insufficient. Typical power densities of the ambient electromagnetic field are situated below $1 \mu \mathrm{W} / \mathrm{cm}^{2}$ [4]. One solution to increase the total amount of harvested energy is to extend the overall antenna area. Two approaches can be distinguished:

Manuscript received June 18, 2012; accepted July 21, 2012. Date of publication August 07, 2012; date of current version August 23, 2012.

N. Degrenne, V. Marian, C. Vollaire, and F. Buret are with Laboratoire Ampere, 69130 Ecully, France (e-mail: nicolas.degrenne@ec-lyon.fr; vlad. marian@ec-lyon.fr; christian.vollaire@ec-lyon.fr; francois.buret@ec-lyon.fr).

$\mathrm{J}$. Verdier is with the Institut de Nanotechnologies de Lyon, 69621 Villeurbanne, France (e-mail: jacques.verdier@insa-lyon.fr).

B. Allard is with Laboratoire Ampere, 69621 Villeurbanne, France (e-mail: bruno.allard@insa-lyon.fr).

Color versions of one or more of the figures in this letter are available online at http://ieeexplore.ieee.org.

Digital Object Identifier 10.1109/LAWP.2012.2212231
- combining several antennas into an antenna association and using a single RF-DC rectifier circuit [5];

- associating a rectifier per antenna and connecting together the dc outputs of the rectifiers [6].

The first approach offers the advantage of supplying a higher energy level to the rectifier and uses the diodes more efficiently. However, for a higher number of antennas in the association, balancing the different signals becomes challenging due to the complex antenna feeds.

The second connection method combines de voltages and currents, being thus insensible to the phase of the input signal. This connection method also offers the advantage of being reconfigurable, as the number of elements in the association can be adapted to the current and voltage requirements of the load. DC output of the rectifiers can be connected in series, parallel, or cascade.

There are situations in which there is a high probability that not all of the rectennas in an association receive the same amount of incident power, like in the case of harvesting energy coming from different directions. In this case, antennas point in different directions, and the dc outputs of each rectenna are associated.

For low available input power, a typical rectenna cell provides very low de voltage, often lower than the minimum input voltage required for powering a dc/dc converter [7], especially for distant emitter or when harvesting ambient energy. By connecting the rectennas in series, the total output voltage is a summation of the individual voltages of each rectenna in the association, increasing the total dc voltage to levels that are more easily exploitable by electronic circuits

Fig. 1 gives the schematic of a three-series-connected-elements rectenna association, as well as its simplified equivalent circuit model.

The total dc voltage and current at load level is given by

$$
\begin{aligned}
V_{\text {out }} & =\sum_{i=1}^{n} V_{i} \\
I_{\text {out }} & =\frac{\sum_{i=1}^{n} U_{i}}{\sum_{i=1}^{n} R_{i}+R_{\text {load }}} .
\end{aligned}
$$

The voltage across one rectenna can be expressed as

$$
V_{i}=U_{i}-I_{\text {out }} R_{i}
$$

As in the case of any generator, if the voltage $V_{i}$ across one of the rectennas becomes negative, that rectenna behaves as an energy receiver, absorbing part of the power generated by the other rectennas in the association. This happens when $U_{i}$ becomes smaller than $I_{\text {out }} R_{i}$, which corresponds to a configuration where the power received by one of the rectennas becomes 


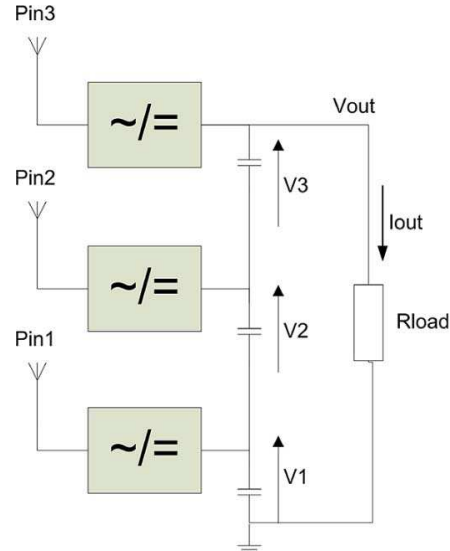

(a)

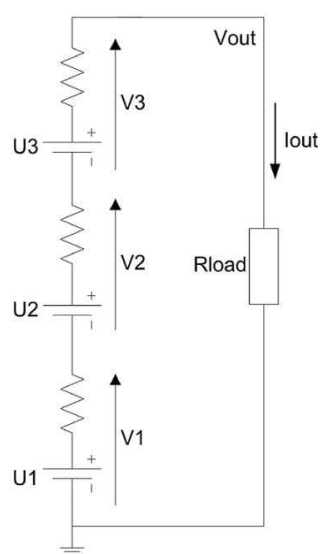

(b)
Fig. 1. (a) Schematic of three-elements series-connected rectenna association. (b) Simplified equivalent circuit model of the association.

considerably small compared to power received by others in the association. The rectenna association is in an unbalanced configuration.

A typical example of application consists of three identical rectifiers that are each connected to one antenna and their $\mathrm{dc}$ outputs are connected in series, as in Fig. 1. In this case, as the rectennas are identical, their internal resistances are also identical: $R_{1}=R_{2}=R_{3}=R$, and this does not vary on incident power. In order to harvest a maximum amount of energy, load resistance has to be equal to source equivalent resistance: $R_{\text {load }}=3 R$. Voltage across rectenna 1 becomes [according to (3)]

$$
V_{1}=\frac{1}{6}\left(5 U_{1}-\left(U_{2}+U_{3}\right)\right)
$$

It is obvious that if rectenna 1 receives considerably less power than the other two rectennas, voltage across it will become negative and it will absorb energy, thus degrading the overall system efficiency.

This letter studies the phenomenon of voltage reversal for three rectennas oriented in different directions. Sections II and III respectively use simulation and experimentations to realize a qualitative and quantitative analysis. Section IV proposes solutions to minimize the detrimental effects of voltage reversal.

\section{Voltage Reversal During Serial Association OF RECTENNAS}

\section{A. Balanced Rectenna Association}

An association of three series-connected rectennas is simulated using Advanced Design System (ADS). The rectennas are based on a single-stage voltage doubler topology using HSMS2850 zero-bias Schottky diodes like previously described in [8]. The association is initially placed in a balanced configuration, and each of the three rectifiers receives a $-5 \mathrm{dBm} R F$ power input.

The static $V-I$ characteristic curve under these conditions is given in Fig. 2(a). Each rectenna contributes to $1 / 3 \mathrm{rd}$ of the total output voltage $V_{\text {out }}$. For low output current values, the output voltage is maximum (above $4 \mathrm{~V}$ ). As the current increases, the voltages decreases because of the increased voltage drops in the

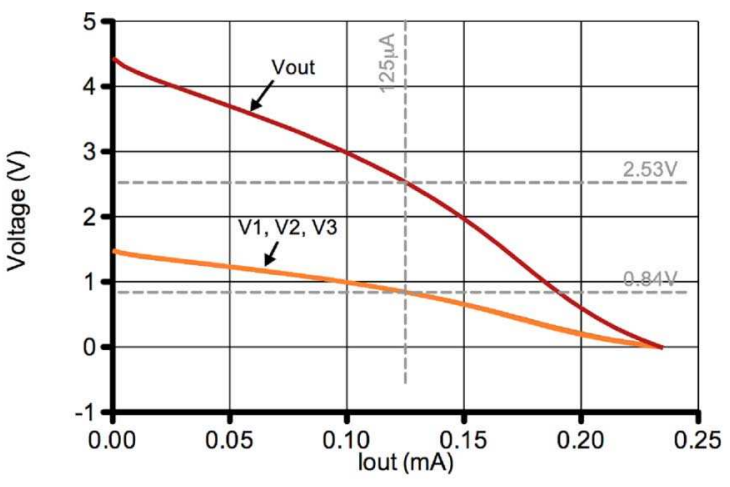

(a)

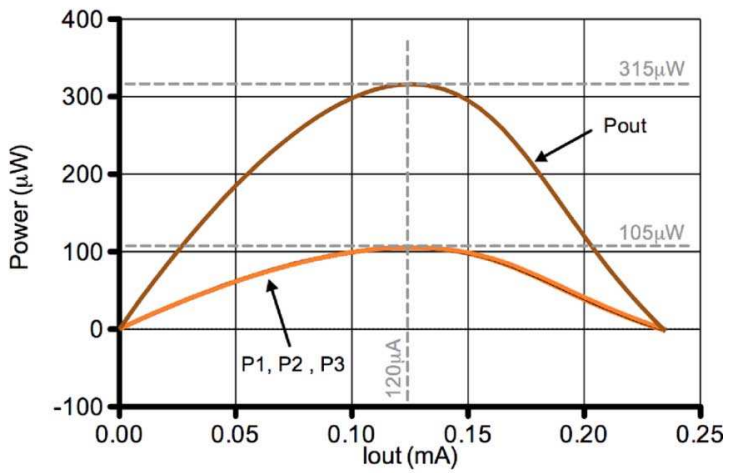

(b)

Fig. 2. Static (a) $V-I$ and (b) $P-I$ curves of a balanced rectenna association (simulation results).

rectenna series equivalent resistances. The short-circuit current is $I_{\mathrm{SC}}=235 \mu \mathrm{A}$ when $V_{\text {out }}=0 \mathrm{~V}$.

The corresponding static $P-I$ characteristic curve is given in Fig. 2(b). The output power is the sum of the individual contributions of each rectenna. The power curves display a noteworthy point, which is the maximum power point (MPP), where the output power is $315 \mu \mathrm{W}$ and the output current is $120 \mu \mathrm{A}$. In most cases, energy will be harvested at this particularly advantageous point. A dedicated power management unit (PMU) can be used to interface the rectenna association and the final load such that the operating point of the association constantly matches the MPP [7].

\section{B. Unbalanced Rectenna Association}

The same association of three series-connected rectennas is simulated in an unbalanced configuration. Rectenna 1 only receives $-20 \mathrm{dBm}$, while the two others receive $-5 \mathrm{dBm}$.

The static $V-I$ curves in Fig. 3(a) show that the voltages at the output of rectennas 2 and $3\left(V_{2}\right.$ and $\left.V_{3}\right)$ are unchanged compared to the previous configuration. The voltage across rectenna 1 is, however, lower than in the previous experience. For this rectenna, the open-circuit voltage is $176 \mathrm{mV}$, and the short-circuit current is around $12.5 \mu \mathrm{A}$.

The static $P-I$ curves in Fig. 3(b) show that rectenna 1 actually absorbs energy from the two other rectennas when the association is operated at MPP $\left(I_{\text {out }}=125 \mu \mathrm{A}\right)$. At this point, the power delivered by rectennas 2 and 3 is $105 \mu \mathrm{W}$, and the power dissipated in rectenna 1 is $24 \mu \mathrm{W}$. The total output power of the association is $186 \mu \mathrm{W}$, which is $11.5 \%$ 


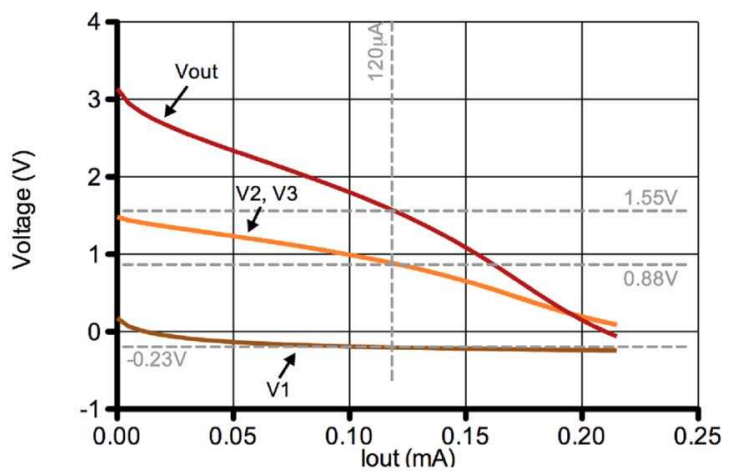

(a)

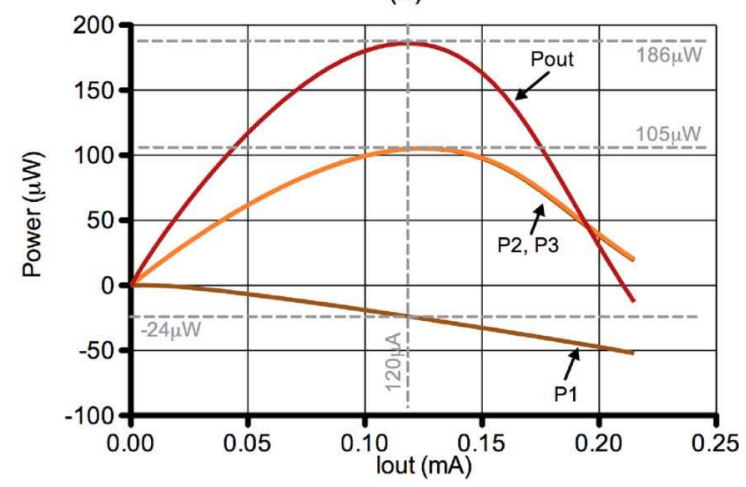

(b)

Fig. 3. Static (a) $V-I$ and (b) $P-I$ curves of an unbalanced rectenna association (simulation results).

less than if only rectennas 2 and 3 were associated. The power dissipated in rectenna 1 corresponds to the conduction losses on the two Schottky diodes in a voltage doubler connection. For higher output current, the losses associated with voltage reversal increase, representing as much as $50 \%$ at $200 \mu \mathrm{A}$.

\section{EXPERIMENTAL RESULTS}

This section investigates experimental energy harvesting from a rectenna association like that simulated in Section II. Each of the three identical rectennas has patch antennas (5-dBi gain) oriented to harvest RF electromagnetic energy from different directions in space. A single electromagnetic power source emits $20 \mathrm{dBm}$ of RF energy at about $2 \mathrm{~m}$ from the association. The emitter has a parabolic antenna with a gain of $18 \mathrm{dBi}$. The orientation of the incident energy on the rectenna association is indicated by $\alpha$ as described in Fig. 4. The whole system is tuned at a central frequency of $2.45 \mathrm{GHz}$.

In a first experiment, the emitter was positioned with an angle $\alpha$ of around $45^{\circ}$, which favors transmission toward rectennas 2 and 3. The load resistor was varied from open-circuit to short-circuit to acquire the experimental static $V-I$ and $P-I$ curves in Fig. 5. At MPP, while rectennas 2 and 3 harvest a positive amount of electrical energy $\left(P_{2}=4.5 \mu \mathrm{W}\right.$ and $\left.P_{3}=3 \mu \mathrm{W}\right)$, rectenna 1 , which is not oriented properly with respect to the emitter, absorbs a fraction of the energy provided by the two other ones $\left(P_{1}=-2 \mu \mathrm{W}\right)$. Rectenna 1 therefore absorbs about $26 \%$ of the energy generated in rectennas 2 and 3 .

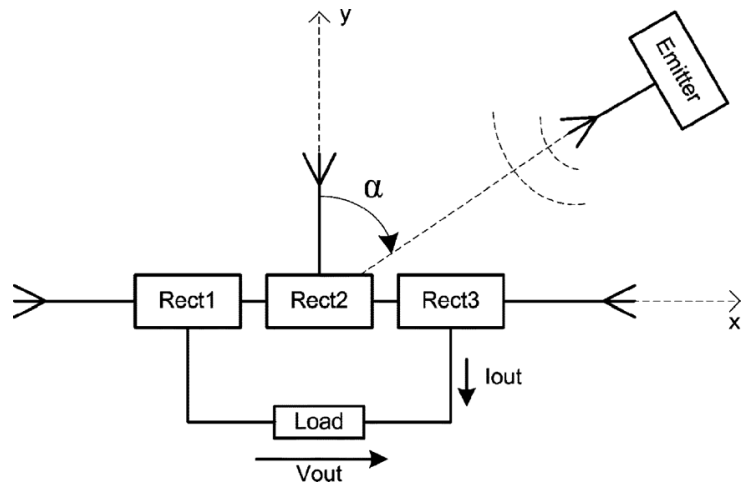

Fig. 4. Setup for the experimental testing of the rectenna association in an unbalanced configuration.

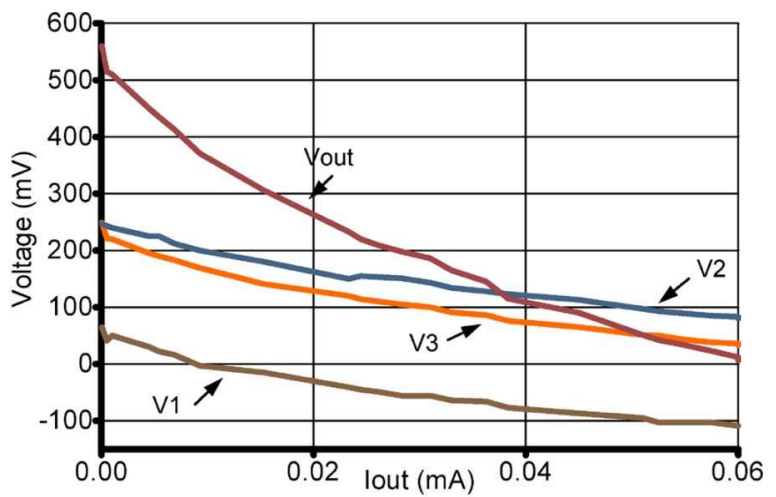

(a)

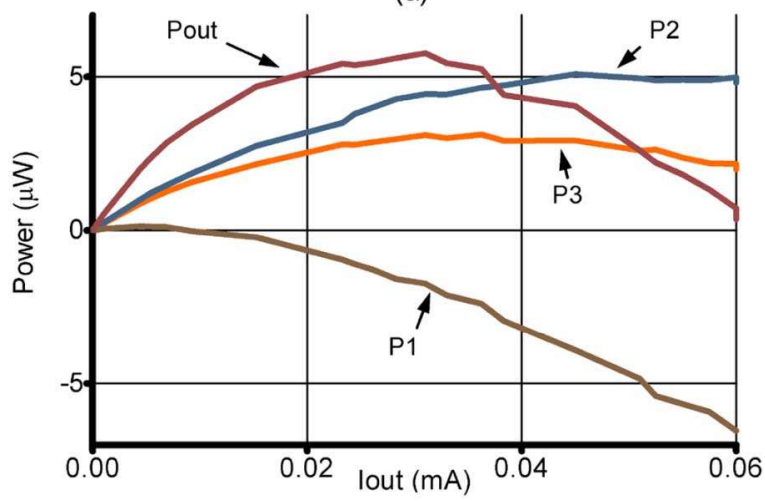

(b)

Fig. 5. Static (a) $V-I$ and (b) $P-I$ curves of an association of multioriented rectennas (experimental results).

A second experiment investigates the effects of the orientation of the emitting power source. Fig. 6 is a representation of the power contribution of each rectenna with changes of $\alpha$. The load was set to $6 \mathrm{k} \Omega$, which corresponds to MPP. For an extreme angle of $90^{\circ}$, the lateral rectenna 3 contributes most to energy production, while the two other rectennas absorb energy. When the emitter is positioned in front of the association (angle of $0^{\circ}$ ), rectenna 2 generates the most amount of power. With this multiorientation harvesting strategy, the output power is always above $5 \mu \mathrm{W}$, which would not be the case for all angles if a single rectenna was to be used. However, the maximum harvested energy is $10 \mu \mathrm{W}$, which is less than if a single rectenna was favorably oriented. 


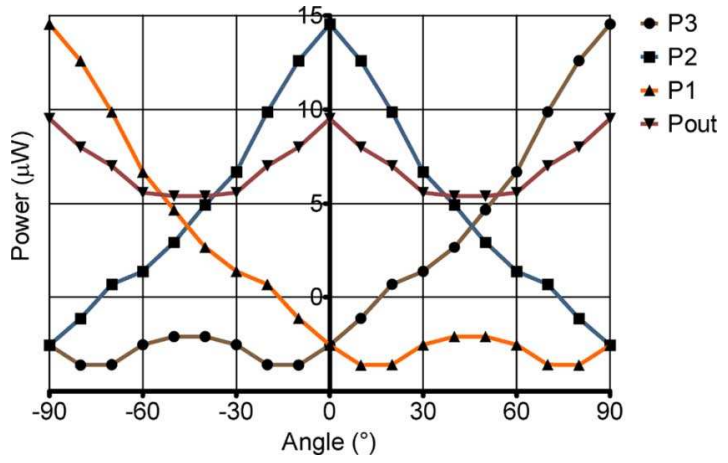

Fig. 6. Power generation of a rectenna association as a function of the electric field incidence angle $\alpha$ (based on experimental results).

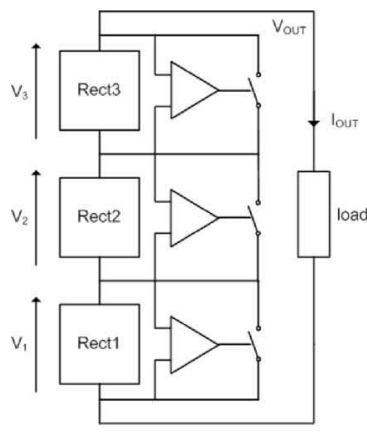

(a)

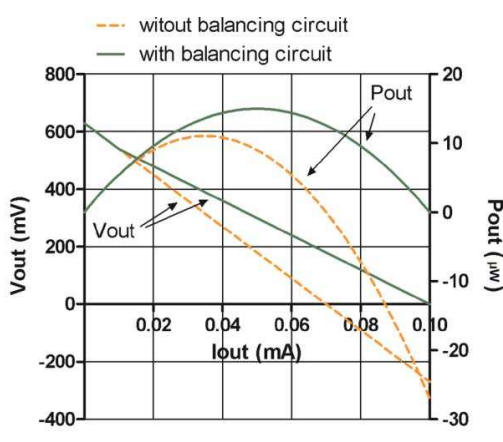

(b)
Fig. 7. (left) Active bypass circuit for rectennas and (right) corresponding simulated $V-I$ and $P-I$ curves.

\section{Discussions}

The simulation and experimental results show that the serial association resulted in a poor global efficiency in case of an unbalanced rectenna association for very low-power applications. A similar inconvenient is encountered when the rectennas are associated in parallel. In this case, the current in a weak rectenna may reverse because of reverse leaking current in Schottky diodes. The following discussion proposes methods to increase the global efficiency of a serial rectenna association.

\section{A. Rectenna Topologies}

The voltage reversal as demonstrated previously takes place when a rectenna association is unbalanced and when the output current is high. In this configuration, the output current exceeds the short-circuit current of the weakest rectennas, and their voltage reverses. The diodes in their output stage conduct continuously, and the voltage reversal corresponds to the forward voltage drop across these diodes. When voltage-doubler rectennas are used, the voltage reversal corresponds to the two diodes of the output stage in series. Other topologies like the monodiode structure would permit to divide the voltage reversal by two and the corresponding power losses by four. When designing a series rectenna association, voltage reversal should be considered as a phenomenon conditioning the design of each rectenna.

\section{B. Active Bypass Circuit}

In order to prevent voltage reversal, a so-called voltage balancing circuit [9] can be used in addition to a main power man- agement unit that sets the operating point of the association to the MPP.

The active bypass circuit is a voltage balancing circuit that enables to decrease the losses associated with voltage reversal and that offers an acceptable compromise between efficiency and ease of implementation. It detects voltage reversal and short-circuit in the concerned rectenna like in Fig. 7 (left). It requires a sensing circuit, a switch, and its driver for each rectenna. Offthe-shelf components (i.e., Texas Instruments TLV3491 comparator) consume currents in the microamp range and can be used if a supply voltage above $1.8 \mathrm{~V}$ is available from the PMU

The principle of the active bypass circuit was tested on the simplified equivalent circuit corresponding to the association of three rectennas in Fig. 1(b) $\left(R_{1}=R_{2}=R_{3}=3 \mathrm{k} \Omega\right.$, $\left.U_{1}=30 \mathrm{mV}, U_{2}=U_{3}=300 \mathrm{mV}\right)$. The static characteristic curves $V-I$ and $P-I$ of the association with and without the active bypass circuit are in Fig. 7. For this simulation, the power consumption of the switches control is neglected, and the switches on-resistance is $1 \Omega$. The $V-I$ curve obtained with the bypass circuit displays a change of slope for an output current of around $10 \mu \mathrm{A}$. The maximum power is from 11 to $15 \mu \mathrm{W}$ without and with the bypass circuit.

\section{CONCLusion}

In this letter, an association composed of three rectennas oriented differently was tested in an unbalanced configuration. Voltage reversal was observed, quantified, and analyzed using both simulation and experimentation at different output currents. The maximum power delivered by the association was decreased by $26 \%$ because of the energy absorption of the reversed rectenna(s). The relative orientation of the electromagnetic emitter influences the relative energy produced by the three rectenna composing the association. Further works will focus on designing a rectenna association that maximizes power generation in nonuniform incident power conditions.

\section{REFERENCES}

[1] V. Marian, C. Vollaire, J. Verdier, and B. Allard, "Potentials of an adaptive rectenna circuit," IEEE Antennas Wireless Propag. Lett., vol. 10, pp. 1393-1396, 2011.

[2] J.-W. Lee and B. Lee, "A long-range UHF-Band passive RFID tag IC based on high-Q design approach," IEEE Trans. Ind. Electron., vol. 56, no. 7, pp. 2308-2316, Jul. 2009.

[3] R. Vullers, H. Visser, B. het Veld, and V. Pop, "RF harvesting using antenna structures on foil," in Proc. PowerMEMS, 2008, pp. 209-212.

[4] H. J. Visser, A. C. F. Reniers, and J. A. C. Theeuwes, "Ambient RF energy scavenging: GSM and WLAN power density measurements," in Proc. 38th IEEE Eur. Microw. Conf., Oct. 2008, pp. 721-724.

[5] V. Marian, C. Vollaire, J. Verdier, and B. Allard, "An alternative energy source for low power autonomous sensors," in Proc. Eur. Conf. Antennas Propag., 2011, pp. 405-409.

[6] G. Monti, L. Tarricone, and M. Spartano, "X-band planar rectenna," IEEE Antennas Wireless Propag. Lett., vol. 10, pp. 1116-1119, 2011.

[7] T. Paing, E. Falkenstein, R. Zane, and Z. Popovic, "Custom IC for ultra-low power RF energy harvesting," in Proc. 24th Annu. IEEE Appl. Power Electron. Conf. Expos., Feb. 2009, pp. 1239-1245.

[8] V. Marian, C. Menudier, M. Thevenot, C. Vollaire, J. Verdier, and B. Allard, "Efficient design of rectifying antennas for low power detection," in Proc. IEEE MTT-S Int. Microw. Symp., Jun. 2011, pp. 1-1.

[9] J. Cao, N. Schofield, and A. Emadi, "Battery balancing methods: A comprehensive review," in Proc. IEEE Veh. Power Propul. Conf., 2008, pp. 3-8. 